\title{
Evaluation of the Hairless Rat as a Model for in Vivo Percutaneous Absorption
}

\author{
Andrea C. Lauer ${ }^{*}$, James T. Elder ${ }^{\ddagger}$, And Norman D. Weiner ${ }^{* x}$ \\ Received August 20, 1996, from the ${ }^{*}$ College of Pharmacy, University of Michigan, 428 Church St., Ann Arbor, MI 48109, and the \\ ${ }^{\ddagger}$ Department of Dermatology and Radiation Oncology (Cancer Biology), University of Michigan, School of Medicine, Ann Arbor, MI 48109. \\ Accepted for publication October $9,1996^{\otimes}$.
}

\begin{abstract}
Percutaneous absorption of topically applied mannitol and progesterone was compared in vivo with the hairless and hairy rat. Urinary excretion and skin concentration profiles after topical application of mannitol demonstrated that hairless rat skin was a "leakier" barrier to percutaneous absorption of polar compounds than was hairy rat skin, independent of formulation. Liposomal, but not aqueous mannitol was retained in hairy rat skin $(>0.5 \%$ after $12 \mathrm{~h}$ ), whereas only negligible amounts were retained in hairless rat skin, regardless of formulation. Progesterone absorption from hydroalcohol and liposomal formulations into hairless rat skin was about five times greater than that in hairy rat skin. Skin delipidization by acetone resulted in a dramatic reduction in the cutaneous barrier to systemic mannitol absorption, which was much more pronounced in hairy than in hairless rat skin. Histological findings of patulous cysts and enlarged, highly vascularized sebaceous glands in the hairless rat suggested that these structures may enhance polar pathways and provide a lipophilic reservoir relative to the fully developed hair follicles of the hairy rat. Collectively, the results document percutaneous absorption differences as a function of animal model, and also suggest that follicular structures make a major contribution to passive percutaneous absorption.
\end{abstract}

\section{Introduction}

The stratum corneum has been described previously as a heterogeneous, dual-natured lipid-keratin matrix, consisting of parallel polar and lipoidal pathways. ${ }^{1-3}$ Besides constituting the major barrier to permeation through the skin, the stratum corneum is also a major pathway for penetration of compounds. ${ }^{4-6}$ The lipid lamellae of the stratum corneum severely restrict penetration of hydrophilic compounds to a remarkably larger degree than that of lipophilic compounds. ${ }^{5}$ Efficient delivery of hydrophilic compounds is dependent on vehide formulation, which may preferentially enhance specific pathways. ${ }^{2}$ The stratum corneum favors the transport of lipophilic compounds, ${ }^{5}$ so it is not surprising that most topical studies have focused on characterizing transport routes for nonpolar, rather than polar drug molecules. Therapeutic applications of peptides and proteins in topical drug delivery have yet to be fully realized.7 Optimal utilization of these exciting new resources will necessitate further characterization of polar routes and development of vehicles to enhance hydrophilic drug delivery.

In addition to the lipid bilayers of the stratum corneum, hair follicles and sebaceous glands have been recognized increasingly as significant routes for penetration of compounds into the skin. ${ }^{7}$ Although hair follicle openings occupy only

\footnotetext{
${ }^{\otimes}$ Abstract published in Advance ACS Abstracts, December 15, 1996.
}

$\sim 0.1 \%$ of the human skin surface area, 8 they are continuous with the viable epidermis, which displays less resistance to polar drug diffusion than does the stratum corneum. ${ }^{5}$ Localized drug delivery to the hair follicle may be a feasible approach to treating several dermatological diseases involving the hair follicle, including acne, rosacea, alopecia areata, and baldness.

Early studies of the follicular pathway were based on the hypothesis that the hair follicle acts as a shunt, resulting in the transport of polar drugs that would not ordinarily be expected to readily traverse the lipid matrix of the stratum corneum. ${ }^{9}$ More recent studies have focused on the possi bility of the pilosebaceous unit acting as a drug reservoir for compounds with varying physicochemical characteristics. ${ }^{10-13}$ Sebum, which flows into the hair follicle from the sebaceous duct, may necessitate a certain degree of lipophilicity for compounds to enter the hair follicle. ${ }^{14}$ Consistent with this concept, topical liposomal formulations localize delivery of carboxyfluorescein and $\alpha$-interferon into sebaceous glands associated with hair follicles. ${ }^{15}$ Interestingly, these studies found that liposomes combined with free drug solution enhance delivery, whereas entrapment may actually hinder the drug delivery process.

The primary difficulty in elucidating the follicular pathway has been the lack of an adequate animal model. Hairless rodents, which are used commonly in percutaneous absorption studies, are not follicle-free. Furthermore, the stratum corneum of hairless rodent skin is typically hyperkeratinized, pilosebaceous units (hair follides and sebaceous glands) are enlarged, and cysts frequently are present in the epidermis, dermis, and hair follicles. ${ }^{16,17}$ These aberrations are also present in the CRL CD hairless rat, which is sometimes described as a "fuzzy" rat because of its sparse, short, broken hairs. ${ }^{18}$ Fuzzy mutants are phenotypically different than their normal siblings in that they have curled vibrissae at birth and a sparse pelage of broken hairs, which after 10 to 15 days may appear slightly fuzzy. Unlike most genetically hairless rodents, this hairless rat strain never develops a wavy coat that rapidly loses hair. Morimoto and co-workers ${ }^{19}$ compared in vitro percutaneous absorption properties of a hairless rat strain and human skin with a wide range of drugs of differing lipophilicities. ${ }^{19}$ Permeability of lipophilic drugs was slightly higher in human skin, whereas permeability of hydrophilic drugs was remarkably higher in hairless rat skin. The contrasting results were attributed to species-specific differences in lipid content and water uptake in the stratum corneum. Hueber and co-workers ${ }^{20}$ concluded that deposition of lipophilic steroids into hairless rat skin was exaggerated due to the presence of numerous hair follicles and sebaceous glands. ${ }^{20}$ The authors ${ }^{21}$ reported that a scarred-skin hairless rat model, ${ }^{21}$ devoid of hair follicles and sebaceous glands, 
provided a reduced depot for lipophilic steroids compared with that provided by intact hairless rat skin. Despite the known aberrations in skin structure and increasing reports of erratic, exaggerated permeability profiles, hairless rodents continue to be popular models that are valued for convenience in dermatological studies.

In these studies, we have investigated mannitol transport in the CRL CD hairless rat and Sprague-Dawley hairy rat upon topical application of aqueous, hydroalcoholic, and liposomal preparations. Mannitol was chosen as the hydrophilic test penetrant because it is a simple, nonmetabolizable, nonionic, and extremely polar molecule. Percutaneous absorption of a highly lipophilic molecule, progesterone, was also examined as an extreme comparison to mannitol deposition. In addition, mannitol and progesterone transport profiles were compared in both rat species after acetone stripping the skin to achieve stratum corneum delipidization. Histological differences in untreated hairless and hairy rat skin, as examined by light microscopy, are also presented. These results indicate that substantial differences in absorption profiles exist be tween hairless and hairy rats, and suggest that follicular structures are major determinants of cutaneous permeability and absorptive capacity.

\section{Materials and Methods}

Materials-D-Mannitol, progesterone, and the buffer salt [HEPES ((4-hydroxyethyl)piperazine-N'-(4-ethanesulfonic acid))] were purchased from Sigma Chemical Company (St. Louis, MO). The unlabeled drug solutions were combined during the formulation process with trace amounts of $>99 \%$ pure $\left[{ }^{14} \mathrm{C}\right] \mathrm{D}$-mannitol or $\left[{ }^{3} \mathrm{H}\right]$ progesterone, which were purchased from NEN (Boston, MA). High purity (> 99 \%) egg phosphatidylcholine (PC) and brain phosphatidylserine (PS) were purchased from Avanti Polar Lipids, Inc. (Birmingham, AL). About $0.1 \mathrm{~mol} \% \mathrm{~d}$ - $\alpha$-tocopherol, which was purchased from Eastman Kodak (Rochester, NY), was added during phospholipid liposome preparation to prevent lipid oxidation. Glyceryl dilaurate (GDL), cholesterol $(\mathrm{CH})$, and polyoxyethylene-10-stearyl ether (POE) were supplied by IGI (Little Falls, NJ). All solvents used were of analytical grade. Ecolite scintillation cocktail was purchased from ICN (Costa Mesa, CA). Male, 6-8-weeks-old CRL CD hairless rats and SpragueDawley hairy rats were purchased from Charles River Laboratories (Wilmington, MA).

Preparation of Formulations-Aqueous solutions containing mannitol were prepared using isotonic HEPES buffer ( $\mathrm{pH} 7.4)$. Hydroalcoholic solutions were prepared with $60 \%$ ethanol (v/v) and mannitol or progesterone, each at $2.5 \mathrm{mg} / \mathrm{mL}$. Phospholipid liposomes comprising PC, $\mathrm{CH}$, and PS at a mole ratio of 1.0: 0.5: 0.1 , were prepared by a conventional film technique as reported by Kirby and Gregoriadis. ${ }^{22}$ Nonionic liposomes comprising GDL, CH, and POE were prepared by a syringe technique at a weight ratio of 58: 15: 27 , as previously described. ${ }^{15}$ "Empty" liposomes were prepared, to which an equal volume of aqueous mannitol solution was added, resulting in a liposome dispersion surrounded by free mannitol in solution. For preparation of progesterone-containing liposomes, radiolabeled and unlabeled progesterone were incorporated into the lipid phase during liposome production to allow for drug intercalation into liposomal bilayers. All liposome formulations contained a final lipid concentration of $50 \mathrm{mg} / \mathrm{mL}$ with either mannitol at $2.5 \mathrm{mg} / \mathrm{mL}$ or progesterone at $100 \mu \mathrm{g} / \mathrm{mL}$.

Deposition Studies-After anesthetizing hairless and hairy rats with sodium pentobarbital in saline $(40 \mathrm{mg} / \mathrm{kg}), 100 \mu \mathrm{L}$ of formulation was applied to a $4.84-\mathrm{cm}^{2}$ dorsal skin site. Hairy rat skin was clipped carefully with an electric clipper $24 \mathrm{~h}$ prior to applying the formulation. Anesthesia was maintained as necessary for the duration of the study. The rats were euthanized at various time points, after which the skin, heart, liver, and kidneys were excised. Hairless rat skin was stripped until a shiny appearance was attained (25 to 30 times), indicating total removal of stratum corneum. The "upper" stratum corneum was defined as tape strips 4-10 (strips 1-3 were excluded due to their retention of drug formulation on the skin surface), whereas the "deeper" stratum corneum was defined as tape strips 11-25. The skin remaining after stripping was designated the "viable skin," consisting of epidermis and dermis. The viable skin was swabbed of excess formulation with a slightly dampened KimWipe. Individual tape strips and viable skin were analyzed for radiolabel by liquid scintillation counting. Hairy rat skin was stripped with heavy-duty clear packaging tape to ensure total removal of hair. Tape strips from hairy rat skin were not assayed because the strips would not distinguish between deposition into stratum corneum and onto the hair shafts remaining post-clipping. As with hairless skin, the residual skin after tape stripping was regarded as the "viable skin." Urine was collected during and upon termination of the study and analyzed by liquid scintillation counting for radiolabel (Beckman LS 6000). Organs were either oxidatively combusted in a tissue oxidizer (Packard I nstruments, Downers Grove, IL) followed by liquid scintillation counting of the resulting condensate, or subjected to extraction by ethanol soaking followed by liquid scintillation counting of the filtered solution. Results were calculated as a percentage of the applied dose, and statistical analysis was performed with Systat software using a two-tailed Student's t test.

Histological Studies-Hairless and hairy rats were euthanized, followed by excision of $4 \mathrm{~cm}^{2}$ dorsal skin sites from each rat. After fixing freshly excised skin in buffered $10 \%$ formalin solution, the specimens were embedded in paraffin and vertically sectioned at 5 $\mu \mathrm{m}$. Sections were hematoxylin- and eosin-stained and examined by light microscopy and photomicrography.

Acetone-Stripping Studies-Hairless and hairy rats were anesthetized as al ready indicated, and then received a direct application of $500 \mu \mathrm{L}$ of acetone in $250-\mu \mathrm{L}$ aliquots by pipette to a $5-\mathrm{cm}^{2}$ dorsal site on each rat. Following this application, a total of $3 \mathrm{~mL}$ of acetone was applied with soaked Kim-Wipe swabs, and gently rubbed into the skin over $\sim 10 \mathrm{~min}$. A site measuring $4.84 \mathrm{~cm}^{2}$ was outlined in the acetone treated area, followed by application of $100 \mu \mathrm{L}$ of $60 \%$ ethanol in water $(\mathrm{v} / \mathrm{v})$, containing progesterone $(2.5 \mathrm{mg} / \mathrm{mL})$ or mannitol $(2.5 \mathrm{mg} / \mathrm{mL})$. After $6 \mathrm{~h}$, the rats were euthanized, the treated skin site was excised, and a laparotomy was performed to remove urine, liver, and blood. The skin was tape-stripped of stratum corneum until shiny and no hairs remained. Viable skin, stratum corneum tape strips, urine, plasma, and liver filtrate were assayed for radiolabel by liquid scintillation counting.

\section{Results}

Deposition Studies-Regardless of formulation and time of sampling, accumulation of radiolabeled mannitol in the urine after topical application was significantly higher in hairless rats than in hairy rats $(p=0.001)$. As shown in Figure 1 , urinary levels of radiolabel in hairless rats approached $1 \%$ after $12 \mathrm{~h}$, whereas urinary levels in hairy rats were al ways $<0.05 \%$. Conversely, $>0.5 \%$ of liposomal mannitol was found in the living skin strata of the hairy rat after $12 \mathrm{~h}$, whereas $<0.1 \%$ of the applied dose was found in the living skin strata of the hairless rat, regardless of formulation (Figure 2). Aqueous solutions resulted in minimal skin deposition in both strains, whereas nonionic and phospholipid liposomal formulations yielded significantly enhanced deposition profiles in hairy rat skin at all time points tested ( $p=$ 0.001 ). Mannitol deposition from phospholipid liposomes was slightly greater than that achieved by nonionic liposomes ( $p$ $=0.03$ ). Formulation effects on mannitol deposition into hairless rat stratum corneum are shown in Figure 3 . After 3 $h$, no differences were noted among formulations, whereas at later time points, nonionic liposomes and hydroalcoholic solutions were superior to aqueous ( $p<0.02$ ) and phospholipid liposomes $(p<0.03)$ in depositing mannitol into the stratum corneum. Although both strains exhibited nearly the same degree of mannitol deposition into the viable skin (i.e., $\sim 0.15 \%$ for both), strikingly greater systemic transport was observed in the hairless rat following topical application of hydroalcoholic solutions ( $>1.5 \%$ versus $0.1 \%$ for hairy rats, $p=0.004$; Figure 4). Regardless of formulation or species, negligible mannitol levels were found in the blood and liver.

After $12 \mathrm{~h}$, distinct differences were also found in progesterone deposition in the viable skin of hairless and hairy rats 

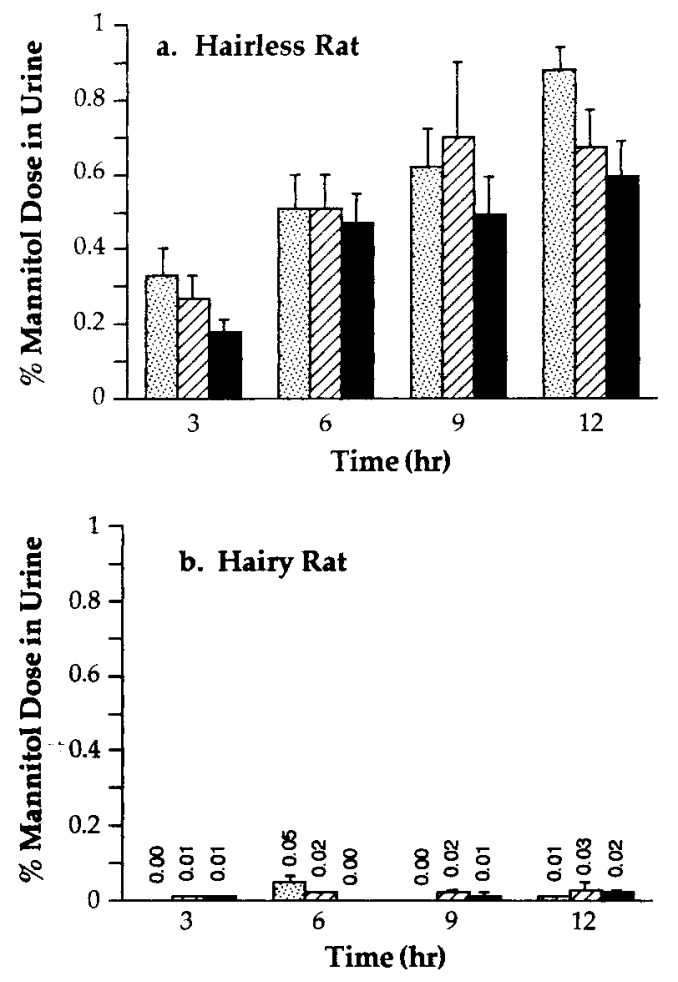

Figure 1-Urinary deposition of mannitol. In vivo deposition of mannitol after topical application of (stippled dots) aqueous solution, (diagonal lines) phospholipid liposomes, or (solid) nonionic liposomes to (a) hairless rat skin and (b) hairy rat skin $(n=3-6$, mean \pm SEM).
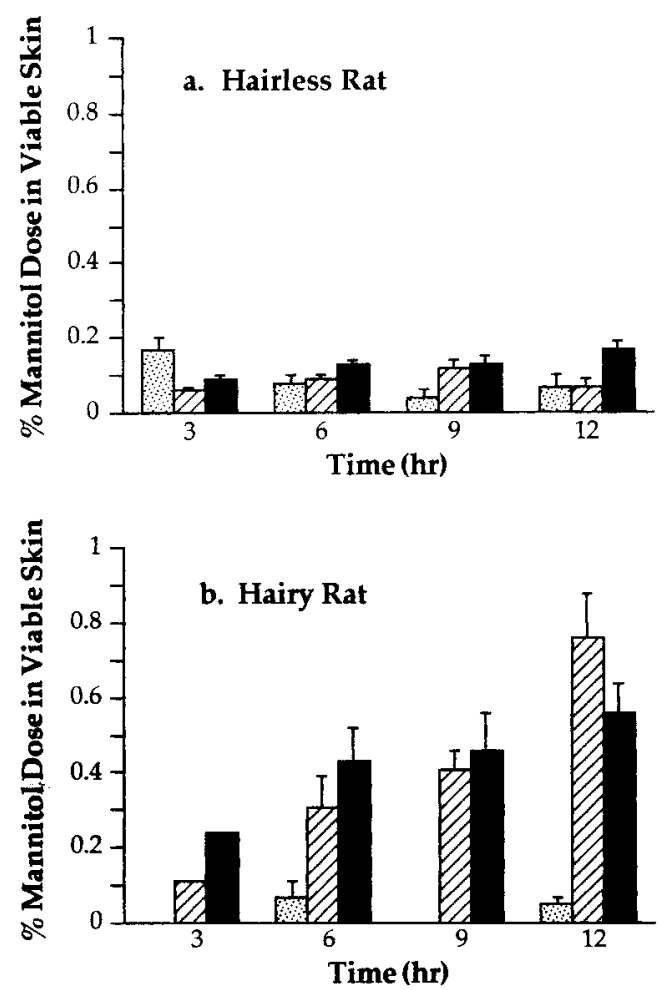

Figure 2-Viable skin deposition of mannitol. In vivo deposition of mannitol after topical application of (stippled dots) aqueous solution, (diagonal lines) phospholipid liposomes, or (solid) nonionic liposomes of mannitol to (a) hairless rat skin and (b) hairy rat skin $(n=3-6$, mean \pm SEM).

(Figure 5). For all formulations, deposition was significantly greater in hairless rat skin $(p<0.02)$, ultimately attaining $\sim 5-6 \%$ of the applied dose. As observed for mannitol,

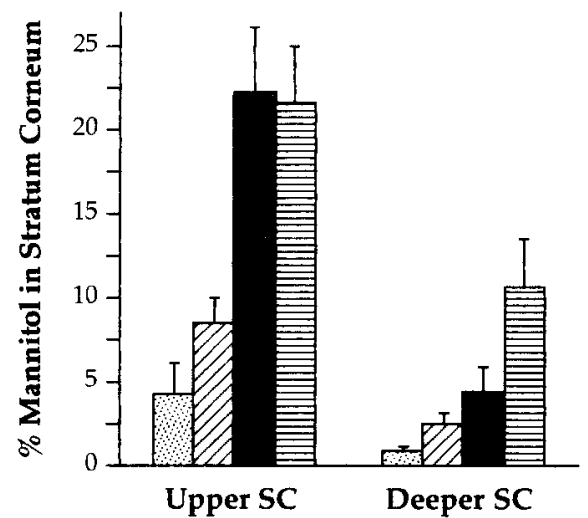

Figure 3-Hairless rat stratum corneum, deposition of mannitol (12 h). In vivo deposition of mannitol into hairless rat stratum corneum (SC) after 12-h topical application of (stippled dots) aqueous solution, (diagonal lines) phospholipid liposomes, (solid) nonionic liposomes, or (horizontal lines) hydroalcoholic solution $(n=3-6$, mean \pm SEM).

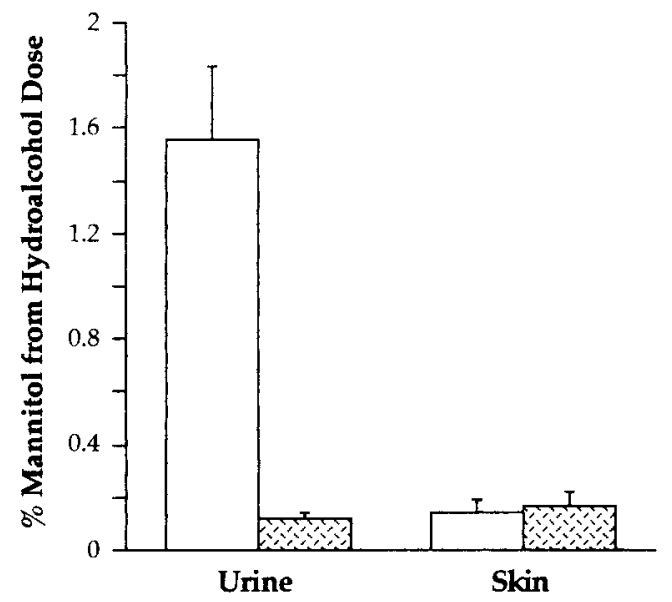

Figure 4-Effect of hydroalcoholic solution (12 h). In vivo deposition of mannitol into (open) hairless rat and (hatch marks) hairy rat urine and skin after 12-h topical application of $60 \%$ ethanol in water ( $/ \mathrm{v} ; n=3$, mean $\pm \mathrm{SEM})$.

systemic transport of progesterone was also significantly greater in hairless rat whether formulated in phospholipid liposomes ( $p<0.01$ ), nonionic liposomes ( $p<0.02$ ), or hydroal coholic solution ( $p<0.01$ ). In comparing formulations used on hairless rat skin (Figure 5a), it was found that the hydroal coholic solution allowed more efficient deposition into viable skin than either liposome formulation ( $p<0.01$ ). In hairless rats, urinary progesterone levels were nearly equivalent $(p>0.05)$, regardless of formulation. After $12 \mathrm{~h}$, hydroalcoholic deposition of progesterone into hairless rat stratum corneum was $\sim 10 \%$ for both the upper and deeper layers, whereas phospholipid and nonionic liposomes achieved $~ 16-$ $17 \%$ and $8 \%$ in the upper and lower stratum corneum, respectively (data not shown).

Histological Studies-Light microscopy revealed gross histological differences between hairy and hairless rat skin. As shown in Figure 6a, hairless rat skin displayed numerous, enlarged sebaceous glands that appeared to be independent of hair follicles, which were themselves atrophic. Numerous cystic structures were present in the epidermis, some of which appeared to be in communication with the skin surface. Many of the enlarged sebaceous glands were filled with lamellated keratin. In contrast, hairy rat skin displayed well-devel oped hair follides clearly associated with smaller sebaceous glands located much deeper in the dermis (Figure 6b). No cysts were apparent in hairy rat skin, and follicle-independent sebaceous glands were observed only rarely, probably as an artifact of sectioning. 

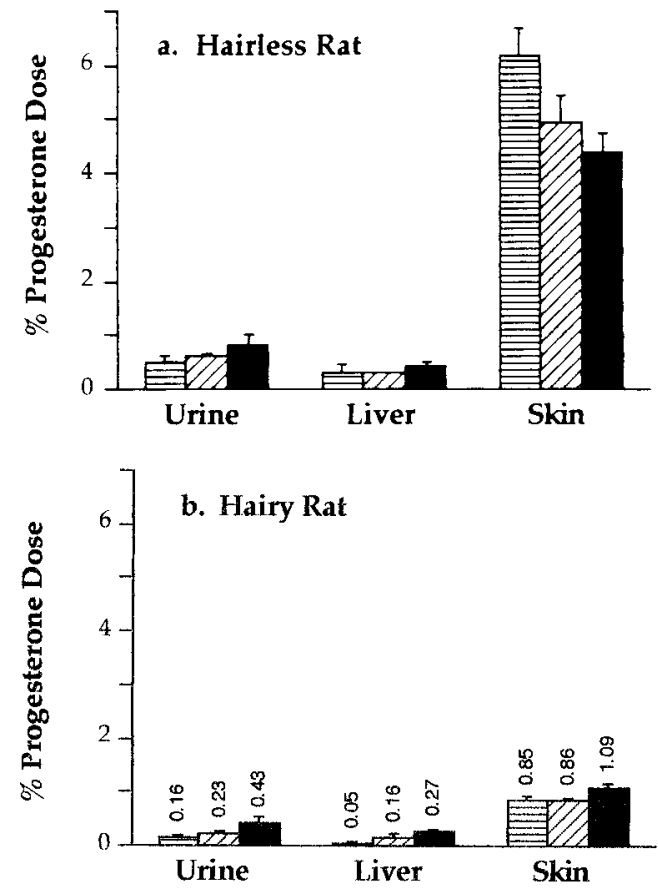

Figure 5-Progesterone deposition from liposomes and hydroalcoholic solution (12 h). In vivo deposition of progesterone into viable skin, urine, and liver after 12-h topical application of (horizontal lines) hydroalcoholic solution, (diagonal lines) phospholipid liposomes, or (lines) nonionic liposomes to (a) hairless rat skin or (b) hairy rat skin $(n=3-4$, mean \pm SEM).

Acetone-Stripping Studies-A 6-h topical application of mannitol after acetone-stripping (Figure 7a) resulted in a remarkable enhancement of systemic mannitol transport (>30\% of the applied dose) through hairy rat skin as compared with hairless rat skin $(p<0.002)$. Mannitol was not detected in the liver or blood, and deposition into the viable skin was $\sim 1.6 \%$ for each species. In contrast, acetone-stripping of hairy rat skin resulted in higher levels of progesterone in the urine $(p<0.005)$ and liver $(p<0.05$; Figure $7 b)$. As observed without stripping, progesterone deposition was significantly lower in hairy rat skin than in hairless rat skin $(p<0.005)$.

\section{Discussion}

Hairless rat skin was far more permeable than hairy rat skin to both progesterone and mannitol, regardless of formuIation (Figures 1 and 2). Collectively, the data suggest that strain differences in skin structure and composition may explain these observations. Our findings of substantial systemic mannitol transport in hairless rat skin failed to match results of past studies of mannitol transport in human skin that indicated a much lower permeability. ${ }^{23,24}$ Hairy rat skin provided a more efficient barrier that largely prohibited mannitol transport into and through the skin, provided that an enhancer system was not present ( $F$ igures $1 b$ and $2 b$ ). These results appear to more closely reflect the human data 23,24 probably because they more closely replicate the human experimental condition than do most previous studies that have been conducted ex vivo and in the presence of organic solvents.

Deposition of mannitol in appreciable amounts into the viable skin of hairy rats occurred only upon topical application of liposome formulations (Figure $2 \mathrm{~b}$ ), suggesting that liposomes preferentially enhance mannitol deposition into hairy rat stratum corneum and eventually into viable skin. Alternatively, fully developed hair follicles of the hairy rat may represent drug depots for li posome-associated mannitol. The
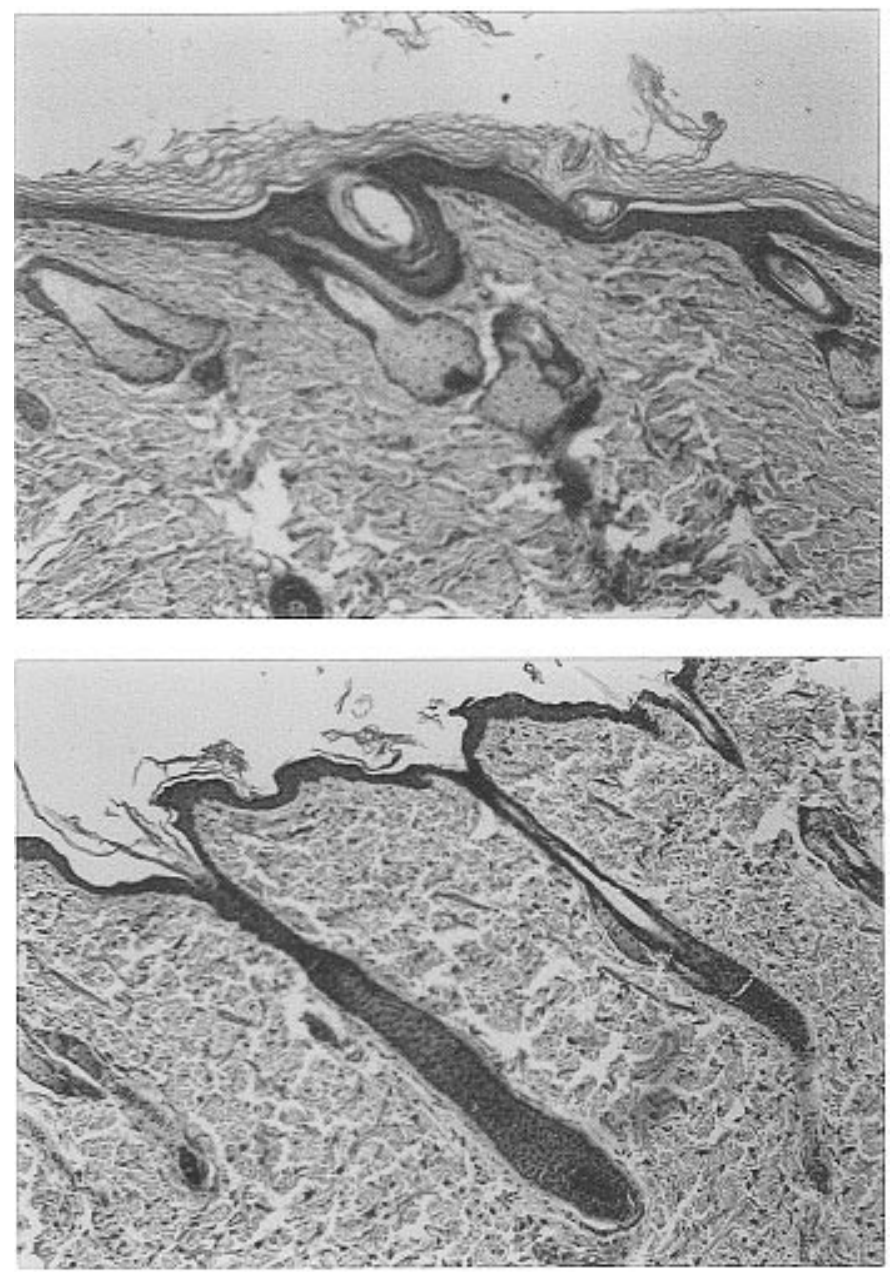

Figure 6-Light microscopy photographs (100X) showing hematoxylin- and eosinstained $5-\mu \mathrm{m}$ vertical skin sections of untreated (a) hairless rat skin and (b) hairy rat skin.

greater deposition of mannitol from phospholipid liposomes was somewhat surprising, as phospholipids only minimally perturb the stratum corneum and therefore possess little intrinsic permeation enhancement activity via this mechanism. ${ }^{25}$ These results would be consistent with a follicular mechanism for delivery, as permeation enhancement effects are generally thought to be mediated via perturbation of the interfollicular stratum corneum. ${ }^{7}$

The major differences in mannitol deposition among formulations were found in hairless rat stratum corneum (F igure 3). Given the known permeability enhancement effects of nonionic liposome components ${ }^{26}$ and ethanol, 24,27 these results are not unexpected. U pon dehydration, GDL melts $(\mathrm{mp}=30$ ${ }^{\circ} \mathrm{C}$ ), which may facilitate the release of $\mathrm{POE}$ from the destabilized bilayers. As a result, both lipids may act independently as enhancers. However, comparison of viable epidermis values showed no differences in deposition among the formulations, indicating that no gradient for transport existed between the stratum corneum and the viable epidermis. The data suggest that upon traversing hairless rat stratum corneum, mannitol was cleared very rapidly from the epidermis and dermis into the systemic circulation. The pharmacokinetics of mannitol, which is entirely renally eliminated, have been described in human and canine studies by Cloyd and co-workers. ${ }^{28}$ Following an intravenous bolus dose of mannitol, the mean distribution half-life in humans is $<5 \mathrm{~min}$, and the elimination half-life is between 1 and 1.5 h. 

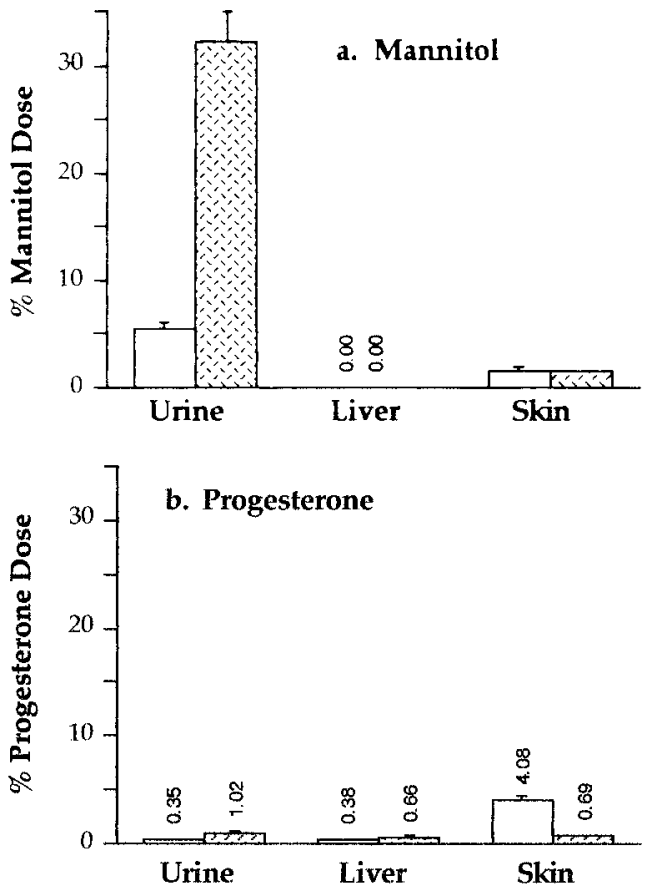

Figure 7-Effect of acetone treatment $(6 \mathrm{~h})$. In vivo deposition of topically applied (a) mannitol and (b) progesterone into viable skin, urine, and liver $6 \mathrm{~h}$ after topical pretreatment of (open) hairless rat and (hatch marks) hairy rat skin with acetone.

As shown in Figure 4, mannitol transport was enhanced slightly in both hairy and hairless rat skin after a 12-h topical application of a $60 \%$ hydroalcoholic solution. Short-chain al cohols are known to increase skin permeability in a reversible manner ${ }^{24,27}$ and are commonly used in topical preparations for this reason. Goates and co-workers ${ }^{24}$ found that ethanol may reorganize or extract lipids and induce conformational changes in the stratum corneum proteins, resulting in a subtle enhancement of mannitol deposition into viable human skin. However, hydroalcoholic solutions did not deliver drug to viable hairy rat skin as efficiently as liposomal dispersions. These results extend the aforementioned similarities between normal human skin and hairy rat skin.

Like mannitol, progesterone also permeated hairless skin more readily than hairy skin. However, the differences between the two strains were less marked than those observed for mannitol, suggesting the hypothesis that nonfollicular pathways may predominate in the transport of lipophilic compounds, whereas follicular pathways predomi nate for more polar compounds. This hypothesis would be consistent with the results shown in Figure 7, which demonstrate significant differences in the behavior of these two compounds as a function of acetone pretreatment.

Acetone has been used in several studies to delipidize the stratum corneum, ${ }^{29-32}$ with the ultimate goal of assessing barrier function and recovery. A single 10-min acetone treatment results in profound impairment of barrier function, with a rapid recovery phase ( $50 \%$ in $6 \mathrm{~h}$ ) followed by a slow recovery phase of 24 to $36 \mathrm{~h}$. Acetone treatment followed by application of progesterone in hydroal coholic solution yielded deposition profiles in both strains that were similar to those obtained without acetone treatment. In contrast, application of mannitol in hydroalcoholic solution yielded remarkably different results, as a large increase in transport was observed as a function of acetone pretreatment in both hairless and hairy strains, with a remarkable $30 \%$ level of systemic absorption in the hairless strain (Figure 7).

The contrasting transport profiles observed for both compounds in hairless versus hairy rat skin may be explicable by the gross differences in skin structure between the two strains (Figure 6). The numerous enlarged and dilated cysts and sebaceous glands of the hairless rat could provide a significant pathway with particularly low resistance to transport of polar compounds, effectively bypassing the normal interfollicular stratum corneum as originally proposed in the "shunt hypothesis" of Scheuplein and Blank.8,9 Considering the rich vasculature surrounding the sebaceous glands, ${ }^{33}$ it is not unreasonable to postulate that the exaggerated systemic transport of mannitol observed in the hairless rat is occurring via such a follicular shunt.

As a plausible explanation for the acetone pretreatment results, consider the possibility that acetone pretreatment has different effects on the permeability properties of the follicular and interfollicular compartments. Thus, I mokawa and coworkers 31,32 found that removal of sebaceous gland lipids from human skin plateaus after $1 \mathrm{~min}$, whereas stratum corneum lipids require much more prolonged acetone treatment to be extracted. 31,32 These results suggest that the stratum corneum lipids are more difficult to extract than are the sebaceous gland lipids. Assuming that our brief acetone treatment condition has similar effects on rat skin and that lipid content is a significant determinant of permeability in both compartments, then such a treatment would be expected to greatly facilitate the absorption of mannitol by providing a more hydrophilic compartment for mannitol transport. If, in contrast, progesterone undergoes absorption primarily via partitioning into stratum corneum lipids, then acetone pretreatment would be expected to have little effect on progesterone transport. This concept would be consistent not only with the observed permeability results, but also with the visual observation that acetone treatment has a much more prominent visible effect on hairless, than on normal mouse skin (data not shown). This hypothesis could be further tested, at least indirectly, by making parallel measurements of permeability, histology, and extracted lipid composition before and after acetone stripping and after "refilling" stripped follicles by application of sebaceous gland or other lipids prior to application of mannitol versus progesterone.

\section{Summary}

A major obstacle in topical delivery studies continues to be the selection of appropriate animal models. The studies presented here emphasize some of the differences in absorption profiles that are animal model-dependent. These results are likely explained by species-specific structural and compositional differences of the stratum corneum and pilosebaceous units, which are important considerations when using animal models for skin permeability studies. Hairless rat skin appears to be an exaggerated model for follicular delivery, but nevertheless suggests that hair follides and sebaceous glands may play a greater role in topical drug delivery than previously assumed. Elucidation of the kinetics of follicular delivery along with optimization of topical formulations are necessary steps toward devel oping drug del ivery systems that target the pilosebaceous unit. The persistence of a vast array of dermatological diseases associated with the pilosebaceous unit hints at the potentially great therapeutic significance of drug delivery to sites associated with this structure.

\section{References and Notes}

1. Yamashita, F.; Yoshioka, T.; Koyama, Y.; Okamoto, H.; Sezaki, H.; Hashida, M. Biol. Pharm. Bull. 1993, 16, 690-697.

2. Yamashita, F.; Bando, H.; Koyama, Y.; Kitagawa, S.; Takakura, Y.; Hashida, M. Pharm. Res. 1994, 11, 185-191.

3. Williams, M. L.; Elias, P. M. Crit. Rev. Ther. Drug Carrier Syst. 1987, 3, 95-122. 
4. Elias, P. M. Arch. Dermatol. Res. 1981, 270, 95-117.

5. Elias, P. M.; Cooper, E. R.; Korc, A.; Brown, B. E. J . Invest. Dermatol. 1981, 76, 297-301.

6. Elias, P. M. J . Invest. Dermatol. 1983, 80, 44-49.

7. Lauer, A. C.; Lieb, L. M.; Ramachandran, C.; Flynn, G. L.; Weiner, N. D. Pharm. Res. 1995, 12, 179-186.

8. Scheuplein, R. J .; Blank, I. H. Physiol. Rev. 1971, 51, 703-747.

9. Scheuplein, R. J . J . Invest. Dermatol. 1967, 48, 79-88.

10. Bidmon H.J .; Pitts J . D.; Solomon, H. F.; Bondi, J . V.; Stumpf, W. E. Histochemistry 1990, 95, 43-54.

11. Fabin, B.; Touitou, E. Int. J. Pharm. 1991, 74, 59-61.

12. Lieb, L.; Ramachandran, C.; Flynn, G.; Weiner, N. J . Invest. Dermatol. 1992, 99, 108-113.

13. Rolland, A.; Wagner, N.; Chatelus, A.; Shroot, B.; Schaefer, H. Pharm. Res. 1993, 10, 1738-1744.

14. Osborne, D. W.; Hatzenbuhler, D. A. In Topical Drug Delivery Formulations; Osborne, D. W.; Amann, A. H., Eds.; Marcel Dekker: New York, 1990; pp 69-85.

15. Weiner, N. D.; Lieb, L.; Niemiec, S.; Ramachandran, C.; Hu, Z.; Egbaria, K. J.' Drug Targeting 1994, 2, 405-410.

16. Inazu, M.; Sakaguchi, T. Lab. Anim. Sci. 1984, 34, 584-587.

17. Hanada, K.; Chiyoya, S.; Suzuki, K.; Hashimoto, I.; Hatayama, I. J . Dermatol. 1988, 15, 257-262.

18. Charles River Laboratories, Wilmington, MA, personal communication, 1996.

19. Morimoto, Y.; Hatanaka, T.; Sugibayashi, K.; Omiya, H. J . Pharm. Pharmacol. 1992, 44, 634-639.

20. Hueber, F.; Wepierre, J .; Schaefer, H. Skin Pharmacol. 1992, 5, 99-107.
21. Illel, B.; Schaefer, H. Acta Derm Venereol (Stockh) 1988, 68, 427-430.

22. Kirby, C. J .; Gregoriadis, G. Biotechnology 1984, 2, 979-984.

23. Barry, B. W.; Bennett, S. L. J . Pharm. Pharmacol. 1987, 39, 535-546.

24. Goates, C. Y.; Knutson, K. Biochim. Biophys. Acta. 1994, 1195, 169-179.

25. Citernesi, U.; Sciacchitano, M. Cosm. Toil. 1995, 110, 57-68.

26. Walters, K. A.; Walker, M.; Olejnik, O. J . Pharm. Pharmacol. 1988, 40, 525-529.

27. Berner, B.; Liu, P. In Percutaneous Penetration Enhancers; Smith, E. W.; Maibach, H. I., Eds.; CRC: Boca Raton, 1995; pp 45-60.

28. Cloyd, J. C.; Snyder, B. D.; Cleeremans, B.; Bundlie, S. R.; Blomquist, C. P.; Lakatua, D. J . Pharmacol. Exp. Ther. 1986, 236, 301-306.

29. Menon, G. K.; Feingold, K. R.; Moser, A. H.; Brown, B. E.; Elias, P. M. J . Lipid Res. 1985, 26, 418-427.

30. Grubauer, G.; Feingold, K. R.; Elias, P. M. J . Lipid Res. 1987, 28, 746-752.

31. Imokawa, G.; Hattori, M. J . Invest. Dermatol. 1985, 84, 282284.

32. Imokawa, G.; Akasaki, S.; Hattori, M.; Yoshizuka, N. J . Invest. Dermatol. 1986, 87, 758-761.

33. Girard, J.; Lafille, C. C. R. Seances Soc. Biol. Fil. 1971, 165, 2085-2091.

J S960350C 